Estudios sobre el Mensaje Periodístico

ISSN-e: $1988-2696$

http://dx.doi.org/10.5209/ESMP.55618

\title{
Uso de las Redes Sociales por las universidades públicas andaluzas. Análisis de contenido de la cuenta oficial de Twitter
}

\author{
José Eduardo Simón Onieva ${ }^{1}$
}

Recibido: 23 de mayo de 2016 / Aceptado: 16 de septiembre de 2016

Resumen. Con la aparición de las Redes Sociales digitales (RRSS) la forma de comunicarse de las universidades con la sociedad de su entorno ha cambiado completamente. Los Gabinetes de Comunicación universitarios se enfrentan a un nuevo reto: adaptarse a una forma de conectar con sus públicos, totalmente diferente. El principal problema ha sido la rapidez vertiginosa con que se han producido dichos cambios. La precipitación puede ser la causa de que ninguna de las instituciones andaluzas haya seguido patrones y estrategias comunes $\mathrm{y}$, cada una, haya tomado sus propias iniciativas. Este estudio ha analizado la cuenta oficial de Twitter de las diez universidades públicas andaluzas durante el mes de febrero de 2013, 2015 y 2016. Se han estudiado alrededor de cinco mil mensajes generados desde los Gabinetes de Comunicación universitarios.

Palabras clave: Universidad; Redes Sociales; Twitter; Comunicación 2.0.

\section{[en] Using Social Networks for the Andalusian public universities. Content analysis of the official Twitter account}

\begin{abstract}
At the time digital Social Networking (RRSS) appeared, the ways of communication of universities with society have changed completely. The Universities Press Offices face a new challenge: to adapt a method which would lead to connect with their audience in a completely different way. The main problem has been the vertiginous speed with by which these changes have happened. Precipitation may be the reason that none of the Andalusian institutions have followed patterns and common strategies and, each one, have taken their own initiatives. This study has analyzed the official Twitter account of the ten Andalusian public universities during the month of February 2013, 2015 and 2016 have been studied about five thousand messages generated from the Universities Press Offices.
\end{abstract}

Keywords: University; Social Networks; Twitter; Communication 2.0.

Sumario. 1. Introducción. 2. Objetivos. 3. Metodología. 4. Las redes sociales y el mundo universitario. 5. Uso de las Redes Sociales por las universidades públicas andaluzas. 6. Análisis de contenido de los mensajes. 7. Discusión y conclusiones. 8. Referencias bibliográficas.

Cómo citar: Simón Onieva, José Eduardo (2017): "Uso de las Redes Sociales por las universidades públicas andaluzas. Análisis de contenido de la cuenta oficial de Twitter", en Estudios sobre el Mensaje Periodístico 23 (1), 631-645.

\footnotetext{
1 Universidad de Almería.

E-mail: jsimon@ual.es
} 


\section{Introducción}

Desde los primeros años de aparición de los Gabinetes de Comunicación, la nota de prensa había sido el único medio utilizado para enviar la información a los medios de comunicación convencionales. El fax fue el gran aliado de los gabinetes durante muchos años. La aparición de Internet y el correo electrónico supuso el ocaso del fax; cambio que mejoraría el trabajo tanto de las redacciones de los medios como de los Gabinetes. Ya era posible enviar textos, fotografías, cortes de audio e incluso vídeos.

Esta revolución tecnológica no quedaría ahí. En poco más de una década aparecen los primeros dispositivos móviles que conectarían a sus propietarios a Internet sin necesidad de cables. A partir de este momento, los usuarios podían estar conectados las 24 horas. Aparecen los blogs, wikis, microblogs y las RRSS. Hay que indicar que las redes sociales no son un fenómeno nuevo. Existen desde los inicios de la Humanidad y sirven para mejorar las relaciones entre los individuos. Son objeto de estudio por parte de la Sociología. Lo novedoso fueron las redes sociales digitales, que permitirían interconectar a millones de usuarios y transmitir la información, de forma casi instantánea, a individuos de cualquier parte del planeta.

Como consecuencia de ésto, los Gabinetes de Comunicación vuelven a sufrir una nueva transformación. Sin abandonar los medios tradicionales, dedicarán parte de sus recursos a esta nueva forma de comunicarse, más directa, rápida y económica. Aunque no todos se adaptaron de forma correcta. Para Paniagua, Gómez y Fernández los Gabinetes de Comunicación universitarios seguían utilizando las RRSS como un "canal de información y no de comunicación" (Paniagua, Gómez y Fernández, 2012: 695).

La sociedad actual está en permanente mutación y los gabinetes deben de evolucionar a la misma velocidad, adaptando sus métodos de trabajo a estos nuevos sistemas tecnológicos. Resulta curioso que, en la década de los noventa, las Universidades españolas fueran de las primeras organizaciones que se adaptasen a Internet. Sin embargo, no se han incorporado con la misma velocidad a la llegada de las redes sociales. Se han visto sobrepasadas por muchas organizaciones, sobre todo, del ámbito privado.

Rodríguez y Santamaría afirman que aunque el 100\% de las universidades españolas estén presentes actualmente en las RRSS, en sus inicios, se mostraron reacias a la incorporación al entorno de la web 2.0. Las redes sociales eran consideradas como elementos dedicados al ocio y muy alejadas del rigor científico (Rodríguez y Santamaría, 2012: 229). Guzmán, por su parte, cree que la principal dificultad para que fuesen adoptadas por el mundo universitario fue por el temor al tremendo cambio que significaba para el ámbito académico, con un enfoque cultural y empresarial diferente (Guzmán, 2012: 134). Se da la paradoja de que, a pesar de estos primeros momentos de indiferencia, el acceso a las RRSS de las universidades españolas fue muy rápido. Otros autores creen que este rápido cambio de actitud se debió a que la mayoría siguió la tendencia de otras instituciones universitarias y, sobre todo, de los campus norteamericanos (Paniagua, Gómez y Fernández, 2012: 694).

El paso de estos iniciales recelos a estar inmersos en las RRSS se produjo de una forma muy rápida y, con frecuencia, precipitada. Rodríguez y Santamaría consideran que las universidades españolas usan las RRSS con "diferente suerte y utilización" (Rodríguez y Santamaría, 2012: 229). En una de las referencias bibliográficas utilizadas en este estudio, sus autores destacarán la existencia de dos usos de Twitter en el mundo universitario: el didáctico y el de la transmisión de la información institucional entre la institución y los estudiantes (Guzmán, del Moral, González y Gol, 2012: 28). 
En este artículo se parte de la premisa de que los Gabinetes de Comunicación universitarios deben ser los servicios encargados de la gestión de las cuentas oficiales en las RRSS. Las cuentas secundarias deberán ser gestionadas por los propios servicios implicados.

\section{Objetivos}

El objetivo del presente artículo es analizar cómo las universidades públicas andaluzas están utilizando las RRSS para conectarse con sus públicos. Para acotar el campo a investigar, nos centraremos sólo en las cuentas oficiales de Twitter de las universidades públicas andaluzas y el espacio temporal a analizar será el mes de febrero.

Se pretende conocer qué tipo de mensajes se incluyen en sus cuentas oficiales de Twitter y medir la respuesta de sus seguidores. Conocer esta respuesta es fundamental para saber si se están aplicando políticas y estrategias comunicativas adecuadas a este nuevo medio. Un mensaje al que los seguidores universitarios han añadido el conocido me gusta o favorito en Twitter viene a indicar que la información es, por lo menos, del agrado del usuario. Si además es compartido o retuiteado entre sus seguidores, eso implica que el mensaje es interesante para que sus amigos o conocidos lo conozcan.

Será interesante conocer a quién se dirigen. Paniagua y Gómez afirman que las universidades cuentan con sus "públicos internos" compuesto por el profesorado (PDI), el personal de administración y servicios (PAS), el alumnado y con "públicos externos", formados por otras administraciones, empresas, antiguos y futuros alumnos, sociedad en general, etc. (Paniagua y Gómez, 2012: 348).

Si en algo destacan las RRSS es en la interactividad entre el emisor y el receptor. Una institución como la universitaria debería perder sus temores a la crítica de sus públicos. Simón defiende la idea que de que las RRSS ofrecen un trato directo y "eso permite que las quejas sobre el funcionamiento puedan llegar de forma más rápida a sus dirigentes. Los responsables de la comunicación universitaria deberían perder sus miedos a la crítica y a la pérdida del control de la información institucional" ( $\mathrm{Si}$ món, 2016: 27). Reina, Fernández y Noguer nos hablarán de "los temores y restricciones institucionales" ante la "inexistencia de una cultura corporativa universitaria" y de la "inmadurez" de dichas universidades en el entorno 2.0 (Reina, Fernández y Noguer, 2012: 132).

En el presente artículo se proponen los siguientes objetivos:

1. Analizar el tipo de mensajes que las Universidades públicas andaluzas incluyen en sus cuentas oficiales de Twitter.

2. Comprobar la respuesta de los seguidores midiendo la interacción.

3. Valorar las estrategias comunicativas que se aplican en las RRSS.

\section{Metodología}

Para tratar de alcanzar los objetivos propuestos, se ha analizado la temática de los mensajes incluidos por las universidades públicas andaluzas durante tres meses correspondientes a cursos académicos diferentes. Para ello se ha aplicado 
la técnica del análisis de contenido. Dicha técnica es considerada por algunos autores como "el método por excelencia de investigación en comunicación" (Antolín, 2012: 20).

Sólo se han analizado las cuentas oficiales de Twitter de las 10 universidades públicas de Andalucía. Como referencias se tomaron los meses de febrero de los cursos 2012/13, 2014/2015 y 2015/2016. Para la obtención de los datos se tuvo en cuenta los obtenidos por Simón en su tesis doctoral y se le han añadido los referentes al curso 2015/16 (Simón, 2016: 257-337).

También se ha tenido en cuenta el estudio de Reina, Fernández y Noguer, autores que realizarían un estudio similar en febrero de 2012 (Reina, Fernández y Noguer, 2012: 132). Por lo tanto el ámbito de la muestra abarcaría a los meses de febrero de cuatro cursos académicos no consecutivos. Aunque se es consciente de que el análisis de contenido de estos autores sólo incluía tres variables y el del autor de este artículo lo amplió a diez, se ha intentado unificarlos y agruparlos para tener una visión lo más unificada posible.

A la hora del análisis se descartaron las cuentas secundarias. Sólo se realizó un seguimiento de la información incluida en las cuentas oficiales de Twitter. Incluir todas las cuentas habría dispersado el ámbito de la investigación y distorsionado el objeto del presente estudio: la información generada por los Gabinetes de Comunicación universitarios que generalmente son los encargados de la gestión de dichas cuentas oficiales.

Se diseñaron tablas Excel con todos los campos establecidos por el análisis de contenido y se contabilizaron todos los mensajes que se adaptaban mejor a cada indicador. Aquellos mensajes que no podían ser catalogados por ninguno de los indicadores de la tabla, se incluyeron en la columna denominada como "varios". Se realizó un seguimiento de las diez instituciones, efectuándose sumas por día y por mes, estableciéndose medias aritméticas y diseñándose gráficas representativas de los datos obtenidos.

También se ha tenido en cuenta el número de mensajes propios y los retuiteados en Twitter. En cuanto a los mensajes retuiteados se ha establecido una división dependiendo de si se trataba de cuentas secundarias de la institución o ajenas a la misma. Para medir la interacción de los usuarios se contabilizaron, por un lado, los mensajes establecidos entre la institución y sus seguidores. Por otro lado, se enumeraron los me gusta o favoritos que los seguidores incluyeron en determinados tuits.

\section{Las redes sociales y el mundo universitario}

Si atendemos a los datos del estudio de TOP-POSITION (2011): "El 97,8\% de los jóvenes españoles que va a comenzar la carrera conoce y utiliza habitualmente Internet, y prácticamente el mismo porcentaje —el 97,7\% - está presente en una o más redes sociales". Carreras, presidente de Top-Position, fue rotundo al afirmar que "las redes sociales están aquí para quedarse. No son una moda pasajera y ya son una parte importante de la vida cotidiana de los jóvenes españoles" (Carreras, 2011)

Según los datos aportados en su estudio por Casero, sólo el 28'8\% de los jóvenes afirman leer diariamente la prensa. Otro dato bastante significativo (en cuanto 
a las diferencias de género): dentro de este grupo que leen diariamente la prensa, el 40 `6\% son hombres y sólo el 19\% son mujeres (Casero, 2012: 3). Si atendemos a los datos aportados por el estudio "Datos y cifras del sistema universitario español. (2012: 26), las mujeres, con un 54,3\%, son mayoría en las Universidades españolas. El cambio en las estrategias comunicativas de los Gabinetes de comunicación universitarios se hace todavía más evidente y urgente.

Los Gabinetes se ven abocados, por tanto, a adaptarse a los nuevos cambios tecnológicos, sociales y comunicativos que se avecinan. Las Redes Sociales comienzan a ser la plataforma de comunicación más rápida y efectiva para llegar a la Comunidad Universitaria y a la sociedad en general. Permiten acceder a un sector de la comunidad universitaria tradicionalmente aislado de la información institucional: el alumnado. Para Simón las RRSS universitarias son mayoritariamente seguidas por alumnos, "sólo queda que el profesorado universitario y el personal de administración y servicios participe, al igual que el alumnado, en las redes de manera activa" (Simón, 2016: 148-149).

Algunos autores defienden la idea de que la visibilidad on-line, la huella o marca digital de las instituciones está condicionada por su presencia en la red. Pero no es suficiente con "estar", hay que complementarlo con una planificación, por parte de los Gabinetes de Comunicación. Hay que garantizar que se está usando el potencial de estas redes "en cuanto a su interactividad con sus públicos, segmentación, viralidad, fidelización y, en síntesis, creación de valor añadido" (Sánchez y Paniagua, 2013: 28).

No se debería pasar por alto un dato importante aportado por Reina, Fernández y Noguer: el horario de actualización de la información incluida en las RRSS. Para estos autores "es diurno y lectivo". La mayoría de los mensajes se concentran a "media mañana y, excepcionalmente, a última hora de la tarde" sin responder a "los hábitos de consumo de estos medios por parte de los usuarios" (Reina, Fernández y Noguer, 2012: 134). Por su parte, Gómez, Roses y Farias llegan a la conclusión de que el $53 \%$ se conectaba varias veces al día a dichas cuentas. La franja horaria con una mayor actividad era entre las 19:00 h. y las 00:00 (Gómez, Roses y Farias, 2012: 3). "Curiosamente, este periodo no suele ser utilizado por los Gabinetes de Comunicación universitarios para incluir su información" (Simón, 2016: 183).

Se hace necesario, por tanto, que los Gabinetes de Comunicación universitarios incluyan su información en la franja horaria más propicia, es decir, en la que están presentes un mayor número de usuarios. Desafortunadamente no coincide con los horarios laborales habituales. Por ello, los responsables de la gestión de las RRSS deberían de utilizar aplicaciones informáticas que permitan la inclusión de información en diferido y programando su difusión en las horas de una mayor audiencia. Aunque existen algunas versiones que se pueden descargar sin coste, las mejores aplicaciones suelen ser de pago. Los responsables de la comunicación universitaria tendrían que plantearse una inversión mínima en la gestión de las redes y abandonar la extendida costumbre de utilizar aplicaciones gratuitas, que cuentan con un limitado número de prestaciones. Dichas aplicaciones ayudarían en el trabajo diario del Community Manager, al aportar estudios de los seguidores, datos estadísticos de uso, etc. facilitando la toma de decisiones posteriores. 


\section{Estudio del uso de las redes sociales de las Universidades públicas andaluzas}

Al efectuar el análisis de todos los mensajes que se incluyen en Twitter, se puede comprobar que, en la mayoría de las ocasiones, la información se actualiza casi a diario (a excepción de los festivos y fines de semana). En el estudio de Reina, Fernández y Noguer se llegaba a la conclusión de que las Universidades andaluzas actualizaban la información en un $60 \%$ de forma diaria, $20 \%$ los fines de semana y otro 20\% los festivos (Reina, Fernández y Noguer, 2012: 134). Durante el periodo estudiado por este artículo, las dos únicas instituciones que han incluido información diariamente, han sido la Universidad de Granada y Sevilla. Indicar que durante los fines de semana y festivos, el número de mensajes incluidos por estas instituciones se redujo significativamente.

En cuanto al volumen de información se detectaron diferencias considerables entre universidades, detectándose importantes variaciones en cuanto al número de mensajes. Existen universidades con mucha actividad informativa y con pobres resultados en cuanto a interacción e instituciones que con una menor cantidad de mensajes consiguen una amplia respuesta de sus usuarios.

A continuación, se adjuntan gráficas y tablas en las que se pueden apreciar el volumen de información que se incluye en las cuentas oficiales de Twitter de las Universidades Públicas andaluzas. En esta ocasión sólo se han tenido en cuenta los mensajes propios. Los mensajes retuiteados serán analizados más adelante.
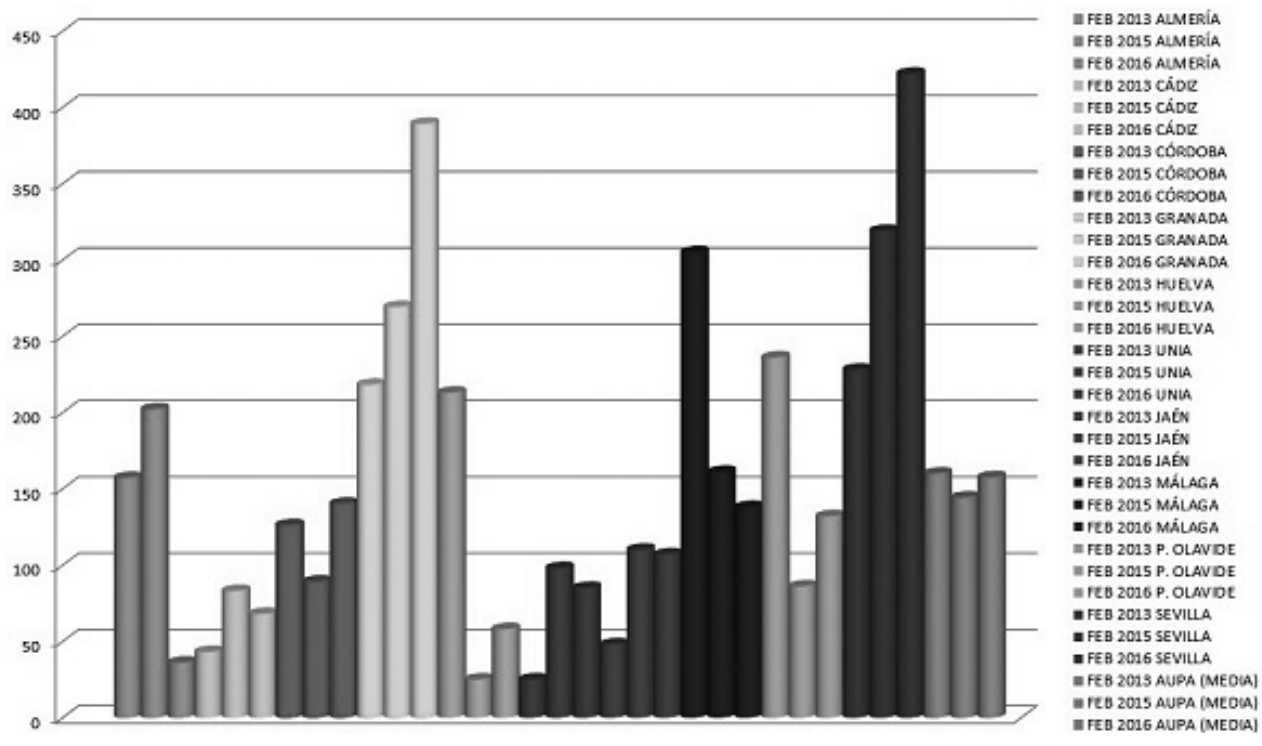

Figura 1. Tuits propios incluidos en las cuentas oficiales. Febrero de 2013, 2015 y 2016. Elaboración propia

En líneas generales desde 2013 a 2016 se produce una estabilización en cuanto al número de mensajes incluidos durante el mes de febrero. Como se podrá ver en la gráfica (Fig.1), esta estabilización del volumen de información incluida en las 
redes se aprecia al establecer la media andaluza. No se traslada por igual a todas las instituciones.

Existen dos Universidades que destacan sobre las demás: Granada y Sevilla. En estas dos instituciones se produce un aumento progresivo del número de mensajes incluidos en las cuentas oficiales. Siendo muy significativo el aumento del último periodo estudiado.

En cambio en otras se produce una bajada o retroceso. Destacarían las caídas estrepitosas de las Universidades de Almería y de Huelva aunque, en esta última, se produce una cierta recuperación en 2016.

Resumiendo, se podría afirmar que durante febrero de 2016 se produce una recuperación generalizada en cuanto al número de mensajes incluidos, superando el retroceso producido en 2015. Destacar también la evolución de la media de tuits diarios. La media andaluza o de $\mathrm{AUPA}^{2}$ se situaba entre los 5,71 tuits de febrero de 2013 y los 5,43 de 2016. Como se puede apreciar en la tabla, existen instituciones que superan dicha media y, por el contrario, otras que están muy lejos de alcanzar estos valores

Si analizamos, por ejemplo, los valores de los meses de febrero de 2015 y 2016 , podemos apreciar que instituciones como la Universidad de Almería sufren un retroceso, en cuanto al número de tuits diarios, del $82,80 \%$. En el extremo opuesto se sitúan la Universidad de Granada y Sevilla que consiguen un incremento del 34,54\% y $27,74 \%$, respectivamente.

Otro dato importante a tener en cuenta sería conocer la interacción de los seguidores. Para ello se contabilizaron tres indicadores diferentes: por un lado, se enumeraron el número de veces que los seguidores se pusieron en contacto con las cuentas oficiales de Twitter para solicitar información, quejarse, felicitar a la institución, etc. Por otro, se contabilizaron los retuits y me gustas/favoritos ${ }^{3}$ que han recibido los mensajes institucionales incluidos en la red.

En la tabla 1 se puede comprobar la actividad de las Universidades andaluzas en cuanto a interactividad. Es posible comprobar el número de mensajes que se establecieron entre los usuarios y las cuentas oficiales. En la columna "Respuestas/Twitter" se contabilizaron los mensajes de los usuarios y las respuestas institucionales. También se puede observar cómo existen universidades con nula actividad en cuanto a intercambio de mensajes con los usuarios y, por el contrario, otras con mucha. Dicha actividad no es constante, constatándose variaciones significativas en los diferentes periodos analizados. La media andaluza sería de 27,9 por mes y la diaria de 0,98 mensajes. Las instituciones con una mayor actividad fueron la Universidad de Granada, con una media de 3,9 mensajes diarios, seguida por la Universidad de Sevilla con 2,26 . En el lado opuesto estarían la Universidad de Jaén con 0,04 y la Universidad Internacional de Andalucía con 0,05.

2 AUPA: Asociación de Universidades Públicas de Andalucía. En el presente trabajo, para abreviar, se utilizará este acrónimo cuando se quiera hablar de las Universidades Públicas andaluzas en su conjunto.

3 A partir de 2015, Twitter sustituiría la estrella que etiquetaba a un mensaje como favorito por el corazón de me gusta. 
Tabla 1. Interacción de los seguidores. Fuente: elaboración propia

\begin{tabular}{|c|c|c|c|c|c|c|c|c|}
\hline UNIVERSIDAD & TUITS & $\begin{array}{l}\text { MEDIA } \\
\text { DIARIA }\end{array}$ & $\begin{array}{c}\text { RESP. } \\
\text { TW }\end{array}$ & $\begin{array}{l}\text { MEDIA } \\
\text { DIARIA }\end{array}$ & RETUITS & $\begin{array}{l}\text { RETUITS/ } \\
\text { MENSAJE }\end{array}$ & $\begin{array}{l}\text { N ME }^{\circ} \text { ME } \\
\text { GUSTA- } \\
\text { FAV }\end{array}$ & $\begin{array}{c}\text { ME } \\
\text { GUSTA/ } \\
\text { MENSAJE }\end{array}$ \\
\hline ALMERÍA FEB 2013 & 157 & 5,61 & 14 & $\mathbf{0 , 5 0}$ & & & & \\
\hline ALMERÍA FEB 2015 & 202 & 7,21 & 45 & 1,61 & 214 & 1,06 & 166 & 0,82 \\
\hline ALMERÍA FEB 2016 & 36 & 1,24 & 6 & 0,21 & 80 & 2,22 & 86 & 2,39 \\
\hline CÁDIZ FEB 2013 & 43 & 1,54 & $\mathbf{0}$ & $\mathbf{0 , 0 0}$ & & & & \\
\hline CÁDIZ FEB 2015 & 83 & 2,96 & 4 & 0,14 & 206 & 2,48 & 119 & 1,43 \\
\hline CÁDIZ FEB 2016 & 68 & 2,34 & 2 & $\mathbf{0 , 0 7}$ & 110 & 1,62 & 74 & 1,09 \\
\hline CÓRDOBA FEB 2013 & 126 & 4,50 & 55 & 1,96 & & & & \\
\hline CÓRDOBA FEB 2015 & 89 & 3,18 & 1 & 0,04 & 212 & 2,38 & 97 & 1,09 \\
\hline CÓRDOBA FEB 2016 & 140 & 4,83 & 5 & 0,17 & 293 & 2,09 & 192 & 1,37 \\
\hline GRANADA FEB 2013 & 218 & 7,79 & 35 & 1,25 & & & & \\
\hline GRANADA FEB 2015 & 269 & 9,61 & 88 & 3,14 & 1196 & 4,45 & 787 & 2,93 \\
\hline GRANADA FEB 2016 & 389 & 13,41 & 159 & 5,48 & 1531 & 3,94 & 1235 & 3,17 \\
\hline HUELVA FEB 2013 & 213 & 7,61 & 4 & 0,14 & & & & \\
\hline HUELVA FEB 2015 & 25 & 0,89 & $\mathbf{0}$ & $\mathbf{0 , 0 0}$ & 99 & 3,96 & 60 & 2,40 \\
\hline HUELVA FEB 2016 & 58 & 2,00 & $\mathbf{0}$ & $\mathbf{0 , 0 0}$ & 181 & 3,12 & 138 & 2,38 \\
\hline UNIA FEB 2013 & 25 & 0,89 & 4 & 0,14 & & & & \\
\hline UNIA FEB 2015 & 98 & 3,50 & $\mathbf{0}$ & $\mathbf{0 , 0 0}$ & 186 & 1,90 & 82 & 0,84 \\
\hline UNIA FEB 2016 & 85 & 2,93 & $\mathbf{0}$ & $\mathbf{0 , 0 0}$ & 149 & 1,75 & 67 & 0,79 \\
\hline JAÉN FEB 2013 & 48 & 1,71 & $\mathbf{0}$ & $\mathbf{0 , 0 0}$ & & & & \\
\hline JAÉN FEB 2015 & 110 & 3,93 & 3 & $\mathbf{0 , 1 1}$ & 484 & 4,40 & 199 & 1,81 \\
\hline JAÉN FEB 2016 & 107 & 3,69 & $\mathbf{0}$ & $\mathbf{0 , 0 0}$ & 627 & 5,86 & 439 & 4,10 \\
\hline MÁLAGA FEB 2013 & 305 & 10,89 & 75 & 2,68 & & & & \\
\hline MÁLAGA FEB 2015 & 161 & 5,75 & 18 & 0,64 & 640 & 3,98 & 443 & 2,75 \\
\hline MÁLAGA FEB 2016 & 138 & 4,76 & 10 & 0,34 & 659 & 4,78 & 524 & 3,80 \\
\hline P. OLAVIDE FEB 2013 & 236 & 8,43 & 71 & 2,54 & & & & \\
\hline P. OLAVIDE FEB 2015 & 86 & 3,07 & 20 & 0,71 & 211 & 2,45 & 98 & 1,14 \\
\hline P. OLAVIDE FEB 2016 & 132 & 4,55 & 25 & 0,86 & 332 & 2,52 & 232 & 1,76 \\
\hline SEVILLA FEB 2013 & 228 & 8,14 & 32 & 1,14 & & & & \\
\hline SEVILLA FEB 2015 & 319 & 11,39 & 82 & 2,93 & 1945 & 6,10 & 1156 & 3,62 \\
\hline SEVILLA FEB 2016 & 422 & 14,55 & 79 & 2,72 & 2176 & 5,16 & 2096 & 4,97 \\
\hline MEDIA AUPA FEB 2013 & 159,90 & 5,71 & 29,00 & 1,04 & & & & \\
\hline MEDIA AUPA FEB 2015 & 144,20 & 5,15 & 26,10 & 0,93 & 539,30 & 3,32 & 320,70 & 1,88 \\
\hline MEDIA AUPA FEB 2016 & 157,50 & 5,43 & 28,60 & 0,99 & 613,80 & 3,31 & 508,30 & 2,58 \\
\hline
\end{tabular}

Por último, hay que indicar que del mes de febrero de 2013 no se cuenta con datos de la interacción con respecto al número de retuits y de me gusta/ favoritos. Como ya se ha indicado, los datos del 2013 y 2015 se han tomado de los incorporados a la tesis doctoral de Simón (2016). Durante el primer periodo 
analizado no se tomaron dichos valores. La imposibilidad de poder recuperar los tuits antiguos que superan la cifra de 3.200 hizo imposible poder recuperar estos datos, sobre todo, en aquellas instituciones con una mayor actividad. Es posible que en el futuro Twitter cambie su forma de operar. De momento, los tuits que superan la cifra de 3.200 se pierden irremisiblemente (Simón, 2016: 370). Como ya se indicó anteriormente, los datos incluidos de 2016 fueron tomados para la realización del presente artículo y se añadieron a los ya obtenidos.

Otros datos interesantes de extraer de la tabla anterior (Figura 2) serían los relativos al número de retuits. Se aprecia un incremento medio del 13,81\%. A nivel andaluz se produce dicha subida pero, como en otras ocasiones, ésta no se cumple en todas las universidades. Si observamos la gráfica anterior (Fig. 4) vemos claramente a dos instituciones que destacan sobre el resto: La Universidad de Sevilla y Granada. En general, todas crecen en número de retuits e interacción, a excepción de la Universidad de Almería, Cádiz e Internacional de Andalucía.

También se incluyen los datos relativos a la media de retuits y de me gusta por mensaje. La media andaluza en febrero de 2015 fue de 3,32 retuits por mensaje y de 1,88 me gusta/favoritos. En el mismo periodo del año 2016 fue de 3,31 y de 2,58 respectivamente, constatándose un descenso en 2016 del $0,30 \%$ en cuanto al número de retuits por cada mensaje subido a la red y un aumento del $37,23 \%$ en cuanto al número de me gusta/favoritos por mensaje.

Como en ocasiones anteriores dichos porcentajes a nivel andaluz no se producen por igual en las diez instituciones andaluzas. Con valores superiores a la media estarían la Universidad de Sevilla, Jaén, Málaga y Granada, en cuanto al número de retuits. Resulta curioso que la institución jienense y la malagueña obtengan mejores resultados que la granadina, contando con un menor número de seguidores. En el lado opuesto estarían el resto. De menor a mayor estarían la Universidad de Almería (que es la institución con peores resultados), Internacional de Andalucía, Cádiz, Córdoba, Pablo de Olavide y Huelva. Indicar que la institución onubense, aunque no superó la media andaluza, obtuvo unos resultados muy cercanos a la media, llegando incluso a superarla en febrero de 2015.

Si analizamos los datos aportados por las siguientes tablas y gráficas de las figuras 2 y 3, se pueden extraer algunas conclusiones interesantes. En cuanto a los retuits de otras cuentas oficiales, se aprecia una disminución generalizada del $17,69 \%$ a nivel andaluz, entre los años 2015 y 2016. Sólo tres instituciones no secundarían esta tendencia: la Universidad de Córdoba, Málaga y Sevilla.

En las siguientes tablas (ver Fig. 2 y 3 ) se incluyen los retuits divididos en dos columnas: una destinada a los retuits pertenecientes a otras cuentas institucionales (RT propios) y los externos (RT ajenos), es decir, los que no pertenecen a la Universidad. 

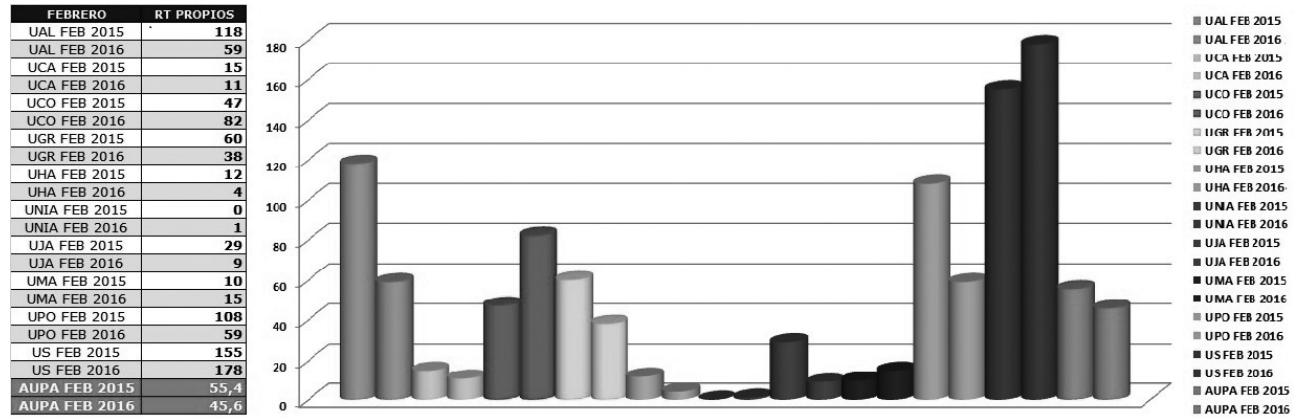

Figura 2. Número de retuits de las cuentas oficiales a otras cuentas de la propia institución. Elaboración propia

En cuanto a los retuits ajenos a la institución, los resultados fueron los siguientes:
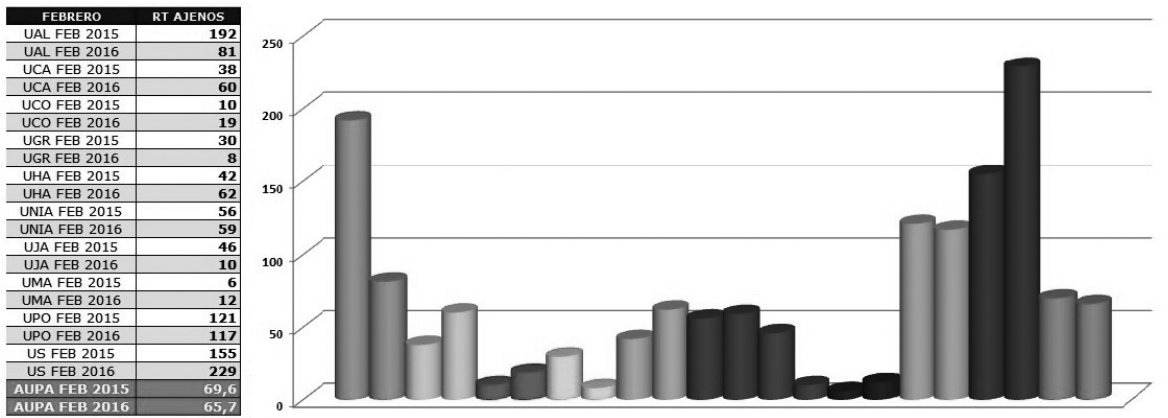

II UAL FEB 2015 TALFEB 2016 - UCA FEB 2015 - UCO FEB 2015 - UCO FEB 2016 $\square$ UGR FEB 2015 IUGR FEB 2016 - UHA FEB 201 UHA FEB 201 - UNIAFEB 2015 - UNIAFEB 2016 - UIA FEB 2015 - UIA FEB 2015 - UMAFEB 2015 - UMAFEB 2016 - UPO FEB 2015 - UPO FEB 2016 - USFEB 2015 - US TEB 2016 IIUPA FEB 2015 IAUPA FEB 2016

Figura 3. Número de retuits de las cuentas ajenas a la institución. Elaboración propia

En cuanto al número de retuits que realizaron de cuentas o personas ajenas a las cuentas institucionales, también se puede apreciar una disminución entorno al 5,6\% menos. En esta ocasión también encontramos instituciones andaluzas en las que se produce el fenómeno contrario, es decir, se produce un aumento (Universidades de Cádiz, Córdoba, Huelva, Internacional de Andalucía, Málaga y Sevilla).

Si observamos los datos de las figuras 2 y 3 se pueden extraer datos interesantes de analizar: ¿Qué Universidad retuitea más sus cuentas secundarias institucionales? y cual lo hace de cuentas no pertenecientes a su institución.

La Universidad de Córdoba, Granada y Málaga difunden más los tuits de sus propias cuentas institucionales que las ajenas. El resto lo difunden al contrario. En la Universidad de Almería, Cádiz, Huelva y Pablo de Olavide se aprecia una mayor tendencia a retuitear tuits ajenos que propios. Dentro de este grupo no hemos incluido a la Universidad Internacional de Andalucía (UNIA), que al ser una institución dedicada a cursos, másteres y postgrados no cuenta con un número de cuentas propias similar al resto (carece de muchos servicios destinados al alumnado, Vicerrectorados, etc. que una Universidad al uso).

Por su parte, en la Universidad de Sevilla se observa, en el primer periodo, un empate en cuanto a los retuits de ambas cuentas y, en el segundo, una diferencia a 
favor de las cuentas ajenas a la institución. A nivel andaluz se puede apreciar una tendencia generalizada a retuitear más los tuits ajenos que los propios.

\section{Análisis de contenido de los mensajes}

A continuación se realizará un análisis de contenido de las cuentas oficiales de las universidades públicas andaluzas. Como ya se indicó anteriormente, se han utilizado los datos aportados por Simón en su tesis doctoral, concretamente los correspondientes a febrero de 2013 y 2015 (Simón, 2016: 257-337). Se le han añadido los referentes a febrero de 2016. Para completar el análisis de contenido, también se incluirán los resultados obtenidos por Reina, Fernández y Noguer, que realizaron un estudio similar en el mes de febrero de 2012 (Reina, Fernández y Noguer, 2012: 132). Señalar que estos últimos autores incluyeron sólo tres indicadores y que en el presente estudio y en la tesis doctoral se utilizaron diez. A pesar de las diferencias en el número de indicadores de ambos estudios, se puede observar que la tendencia es muy similar en cuanto a las temáticas más usadas en Twitter por las Universidades Públicas andaluzas.

En este apartado se incluyen los resultados del análisis de contenido de los mensajes incluidos, con la pretensión de comprobar qué tipos de mensajes predominan en las RRSS de las universidades andaluzas.

Como ya se ha indicado en el apartado de metodología, el presente estudio se dividió en tres fases: la primera comprendió el mes de febrero de 2013, la segunda, incluyó el mismo periodo pero de 2015 y, la última, del año 2016. Los criterios que se han utilizado en la clasificación temática se han tomado de los que incorporó Simón en su tesis doctoral (Simón, 2016: 48-50).

Por temáticas, la más usada en los tres periodos fue la dedicada a Cursos, Conferencias, Jornadas, exposiciones, etc., seguida de la información docente, académica y de política universitaria. Por el contrario, las menos usadas — de menor a mayor - fueron las dedicadas a empleo, solidaridad o voluntariado y las destinadas a información al alumnado.

Por porcentajes, los mensajes dedicados a cursos, jornadas y conferencias se situaron entorno al 32,34\% del total de mensajes incluidos por las Universidades públicas andaluzas, seguido de los relativos a información académica, docente o de política universitaria, que se situaría en un $18,73 \%$ de los mensajes y de los clasificados como varios, en el 15,41\%.

Si comparamos estos resultados y los equiparamos a los indicadores utilizados por el estudio de Reina, Fernández y Noguer vemos que los resultados en febrero de 2012 son similares, con la limitación de los pocos indicadores que utilizaron. Estos autores llegaron a la conclusión de que las instituciones andaluzas mayoritariamente difundían noticias relacionadas con la institución el 80\%, temas de docencia, horarios y becas el $70 \%$ e información relativa a cursos y eventos el $70 \%$ (Reina, Fernández y Noguer, 2012: 135). 


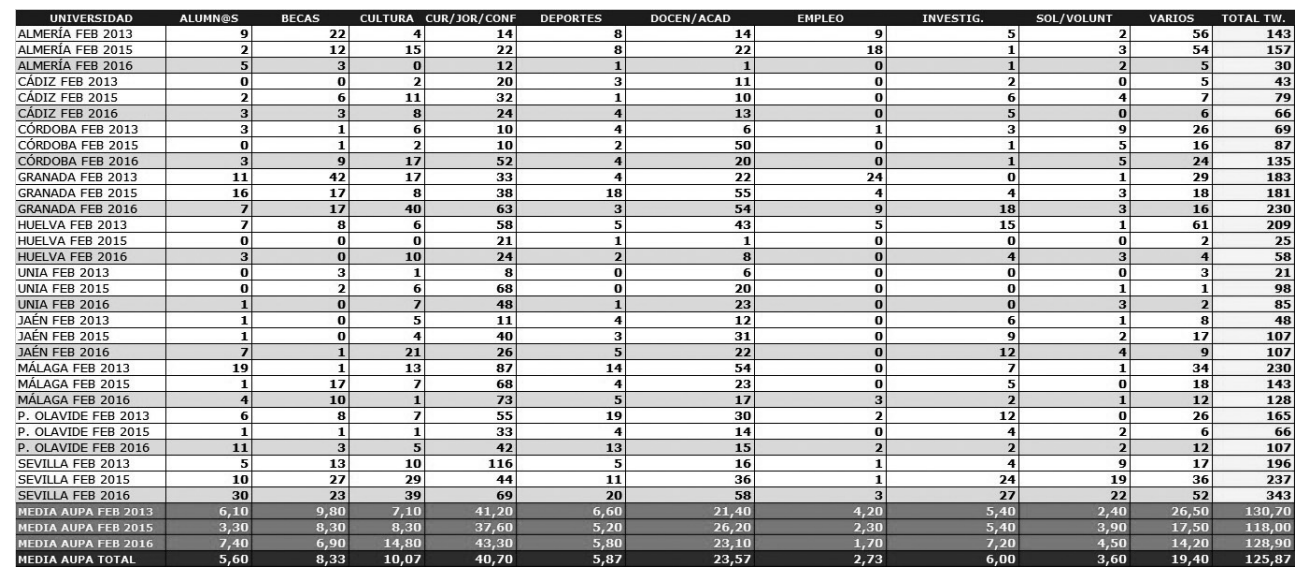

Tabla 2. Análisis de Contenido: número de mensajes distribuidos por temáticas.

Elaboración propia

\section{Discusión y conclusiones}

A la vista de los datos aportados en el presente estudio, se puede llegar a diferentes conclusiones, dependiendo de los datos analizados. Tal y como indica Simón, las instituciones universitarias utilizan las redes sociales "como un nuevo medio de publicitarse y de mejorar su imagen corporativa, no preocupándose, de igual manera, por conectar e interactuar con sus usuarios" (Simón, 2016: 374). De la misma opinión es Guzmán. Esta autora afirmará en su tesis doctoral que “...las universidades utilizan las redes sociales para promocionarse ellas mismas y generar actividad en la comunidad educativa a través de la participación de eventos" (Guzmán, 2013: 298).

Veas defiende la idea de que las universidades dedican sus esfuerzos en "gestionar las comunicaciones hacia el público externo", olvidándose de la comunidad universitaria y de los alumnos en particular que "requieren de calidad en la comunicación definida para ellos" (Veas, 2014: 184). Hay que aclarar que esta autora no se refería a la comunicación en las RRSS sino a la comunicación universitaria en general y la chilena en particular. Por su parte Paniagua, Gómez y Fernández creen que el principal problema de la comunicación universitaria en las RRSS es que los gabinetes de comunicación "....adoptaron Internet como un canal de información y no de comunicación” (Paniagua, Gómez y Fernández, 2012: 695). Sánchez y Paniagua, por su parte, cuando se refieren a la comunicación organizacional en las redes sociales digitales afirman que “...mantiene los principios básicos de la comunicación tradicional, pero desarrolla su actividad en un nuevo espacio comunicativo" (Sánchez y Paniagua, 2013: 30), espacio que es muy diferente a los medios tradicionales, con unas características y peculiaridades propias.

En general, los mensajes de los Gabinetes de Comunicación en las RRSS se han quedado completamente obsoletos. Orosa afirma tajante que dichos mensajes tienen que cambiar porque ha evolucionado el público y las fuentes a los que iban destinados. El receptor de los mensajes se convierte, a su vez, en emisor. Esta autora defiende la idea de que la información de los gabinetes no puede ser "unidireccional 
y asimétrica, sino que diferentes personas e instituciones podrán hablar de ella y conversar con ella" (Orosa, 2009: 9).

Otra de las conclusiones a la que se llega tras el análisis de los datos expuestos anteriormente es que incrementar el número de mensajes no implica un aumento de la interacción por parte de los usuarios. Los Gabinetes deberían de plantearse analizar la información que incluyen en las redes y crear mensajes propios para éstas, diferentes a los que envían a los medios de comunicación. También deberían tener presente que la red social Twitter tiene unas características propias que la diferencian de otras plataformas. No sólo nos referimos a los famosos 140 caracteres, sino a la propia idiosincrasia de esta red y a las características de sus seguidores. Conocer a fondo esta red social nos puede permitir mejorar la forma de llegar a sus públicos que, en el caso que nos ocupa, sería la comunidad universitaria. Esta regla podría ser aplicada al resto de plataformas. Se hace necesario que los mensajes se adapten a cada red social, a la cuenta y al tipo de seguidores que predominan.

Autores como Rodríguez creen que lo más importante de las RRSS es crear diálogo con los usuarios y que éste sea bidireccional. Afirma que "Cuando no se publica sin más, y se intenta conversar, siempre se termina obteniendo una respuesta positiva por parte del usuario. El diálogo, como base de un flujo de comunicación en los dos sentidos, es primordial" (Rodríguez, 212: 157).

Reina, Fernández y Noguer llegaron a la conclusión de que las Universidades no utilizaban de forma adecuada las redes sociales, que no le sacaban el rendimiento adecuado. Afirman que "...las universidades andaluzas usan las redes sociales, pero que la comunicación a través de estas herramientas no es la mejor ni la más provechosa" (Reina, Fernández y Noguer, 2012: 139).

En referencia al tipo de mensajes a incluir en las RRSS universitarias, Simón llegó a la siguiente conclusión: "Los Gabinetes de Comunicación universitarios deberían de plantearse analizar la información que incluyen en sus Redes Sociales y adaptarlos a la características de los mensajes que demandan sus seguidores, a los horarios de uso y entablar un diálogo bidireccional con sus públicos. Inundar de mensajes la red sin un contenido atractivo es tan perjudicial como no actualizar dichos contenidos" (Simón, 2014: 158).

Otro aspecto a reflexionar sería si los resultados que se han obtenido en este artículo, sobre el análisis de contenido, son extrapolables a otras instituciones universitarias no andaluzas. Podríamos estar ante una tendencia localizada a nivel regional y que en el resto del país fuese diferente. Parece ser que no. Que estamos ante una costumbre o tendencia generalizada en el mundo de la comunicación universitaria en las RRSS. Para apoyar esta reflexión podríamos utilizar los datos de Guzmán. Esta autora analizó a 519 universidades de todo el mundo. Llegó a una conclusión similar a la del autor de este artículo: los contenidos más utilizados en las cuentas oficiales de Twitter fueron los relativos a "promoción de los servicios, publicaciones sobre su imagen corporativa y publicaciones dirigidas a la comunidad educativa" (Guzmán, 2013: 315). Por tanto, podríamos encontrarnos ante una tendencia generalizada que se extendería más allá del espacio andaluz. En conclusión, podríamos resumir que la comunicación universitaria en las RRSS se acercaría más a la comunicación tradicional: la destinada a los medios convencionales. Los Gabinetes de Comunicación universitarios centrarían todos sus esfuerzos en autopublicitar a su institución. Interactuar con sus seguidores quedaría relegado a un segundo plano. 
Por último, indicar que los resultados de este estudio están localizados al ámbito universitario andaluz y a un momento determinado en el tiempo. Sólo se ha analizado el $12,04 \%$ de las universidades españolas y el $20 \%$ de las públicas. Posiblemente los resultados que se han obtenido habrían sido diferentes de haberse seguido otras líneas de investigación o aplicado una metodología diferente. Sería interesante que, en un futuro próximo, se pudiera relacionar el caso andaluz con el de otras universidades españolas o europeas. Quedan abiertos, por tanto, nuevos caminos que merecerían ser investigados en los próximos años.

\section{Referencias bibliográficas}

Antolín Prieto, Rebeca (2012): Youtube como paradigma del vídeo y la televisión en la WEB 2.0. Tesis Doctoral. Universidad Complutense de Madrid. http://eprints.ucm.es/16111/1/ T33817.pdf [Consulta: 18 de abril de 2016]

Carreras Lario, Ricardo (2011): "Estudio Jóvenes Universitarios y Redes Sociales-Top Position". Top-Position. Consultora de Comunicación Digital.

http://posicionamientoenbuscadoreswebseo.es/estudio-jovenes-universitarios-y-redessociales/. [Consulta: 18 de abril de 2016]

Casero Ripollés, Andreu (2012): "Más allá de los diarios: el consumo de noticias de los jóvenes en la era digital". Revista Comunicar. En:

http://www.revistacomunicar.com/pdf/preprint/39/C39-15-PRE-15671-CASERO-es.pdf [Consulta: 18 de abril de 2016]

Guzmán Duque, Alba Patricia.; Del Moral Pérez, María Esther; y González Ladrón De Guevara, Fernando (2012): "Usos de Twitter en las universidades iberoamericanas". Relatec. Revista Latinoamericana de Tecnología Educativa, 11 (1), en: http://relatec.unex.es/article/viewFile/845/635 [Consulta: 20 de febrero de 2016]

Guzmán Duque, Alba Patricia (2013): Factores críticos de éxito en el uso de las Redes Sociales en el ámbito universitario. Tesis doctoral. Universidad Politécnica de Valencia. https://riunet.upv.es/handle/10251/28212 [Consulta: 22 de enero de 2016]

Gómez Aguilar, Marisol; Roses, Sergio; y Farias Batlle, Pedro (2012): "El uso académico de las redes sociales en universitarios". Revista Comunicar. DOI: 10.3916/C38-2011-03-04.Editorial Preprint.- http://www.revistacomunicar.com/pdf/preprint/38/14-PRE-13426. pdf [Consulta: 25 de abril de 2016]

García Orosa, Berta (2009): Gabinetes de comunicación on line: Claves para generar información corporativa en la red. Sevilla, Comunicación Social, Ediciones y Publicaciones.

Paniagua Rojano, Francisco Javier y Gómez Calderón, Bernardo J. (2012): "Hacia la comunicación 2.0. el uso de las redes sociales por parte de las Universidades españolas". Revista de Comunicación y Tecnologías emergentes Icono 14, 10 (3), 346-364- http:// dx.doi.org/10.7195/ri14.v10i3.473 [Consulta: 1 de marzo de 2016]

Paniagua Rojano, Francisco Javier; Gómez Calderón, Bernardo J.; y Fernández Sande, Manuel (2012): "La incorporación de los departamentos de comunicación de las universidades españolas al entorno digital. Un análisis cuantitativo". Estudios sobre el Mensaje Periodístico, 18, Núm. especial noviembre (2012), 691-701. http://revistas.ucm. es/index.php/ESMP/article/viewFile/40948/39198 [Consulta: 19 de abril de 2016]

Ministerio de Educación, Cultura y Deporte (2015):. "Datos y cifras del sistema universitario español. Curso 2014-2015, en: 
.http://www.mecd.gob.es/dms/mecd/educacion-mecd/areas-educacion/universidades/ estadisticas-informes/datos-cifras/Datos-y-Cifras-del-SUE-Curso-2014-2015.pdf [Consulta: 1 de febrero de 2016]

Reina Estévez, Jesús; Fernández Castillo, Iris; y Noguer Jiménez, Álvaro (2012): “El uso de las redes sociales en las universidades andaluzas: el caso de Facebook y Twitter". Revista Internacional de Relaciones Públicas, II, n 4, 123-144. http://dx.doi.org/10.5783/RIRP-4-2012-06-123-144 [Consulta: 23 de abril de 2016]

Rodríguez Fernández, Óscar (2012): Curso de Community Manager. Madrid, Ediciones Anaya Multimedia.

Rodríguez Ruibal, Antonio y Santamaría Cristino, Patricia (2012): “Análisis del uso de las redes sociales en Internet: Facebook y Twitter en las Universidades españolas". Revista de Comunicación y Tecnologías emergentes Icono 14, 10 (2), 228-246, en: http://dx.doi.org/10.7195/ri14.v10i2.198 [Consulta: 23 de abril de 2016]

Sánchez González, María y Paniagua Rojano, Francisco Javier (2013): “Estrategias de comunicación 2.0 en asociaciones profesionales. Estudio del caso de los Colegios Oficiales de Médicos en España". Revista Mediterránea de Comunicación, 4 (1), 21-51, http://rua.ua.es/dspace/bitstream/10045/27375/1/ReMedCom_04_01_02.pdf [Consulta: 27 de abril de 2016]

Simón Onieva, José Eduardo (2014): "El uso de las Redes Sociales en el ámbito de la comunicación universitaria andaluza". Revista Internacional de Relaciones Públicas, IV, 8, 139-160. http://dx.doi.org/10.5783/RIRP-8-2014-08-139-160 [Consulta: 15 de abril de 2016]

Simón Onieva, José Eduardo (2016): Gabinetes de Comunicación universitarios y redes sociales. Estudio del uso de las Redes Sociales por las universidades públicas andaluzas. Tesis doctoral. Universidad de Almería. https://www.educacion.gob.es/teseo/irGestionarConsulta.do [Consulta: 27 de abril de 2016]

Veas Yáñez, Claudia (2014): Política comunicacional en las universidades chilenas. Tesis doctoral._ Universidad de Málaga (Ed.).

http://riuma.uma.es/xmlui/bitstream/handle/10630/9542/TDR_VEAS_YANEZ. pdf? sequence $=1$ [Consulta: 27 de marzo de 2016]

José Eduardo Simón Onieva es doctor por la Universidad de Almería, jefe de negociado del Servicio de Gestión de Recursos Humanos de la Universidad de Almería. Actualmente pertenece a dos grupos de investigación de la Universidad de Almería: CySoc Comunicación y Sociedad de la UAL y Estudios del Tiempo Presente. 